\section{The Scanning Electron MACROscope}

\author{
Vernon E. Robinson, JEOL USA, Inc.
}

A good scanning electron microscope is not measured only by its ultimate resolution but often by its flexibility as well. In the real world not many samples require their surface to be examined at $100,000 \mathrm{X}$ to $300,000 \mathrm{X}$. Other real considerations are its analytical capabilities, such as EDS and WDS for chemical analyses and its flexibility to handle odd samples or odd applications, such as STEM imaging, electron channeling, cathodoluminescence, etc.

Have you ever wished that the lowest magnification of your SEM wasn't $10 \mathrm{X}$ or $20 \mathrm{X}$, but rather $5 \mathrm{X}$ or $2 \mathrm{X}$ or $1 \mathrm{X}$ or even lower? This can be especially useful in a forensic application where large samples are common and cutting them or using other destructive techniques would render the sample useless in court.

If you are a user of any of the following JEOL SEM's you can achieve images down to approximately $1 / 2 X$ magnification by running the lenses of your microscope in an unconventional manner. The instruments capable of this task are: all JSM-840 series, JSM-840F, JSM-6300. JSM-6400, and JSM-6600.

To get a very low magnification view of a sample like that in Fig. 1 you would set the microscope up in the electron channeling mode (see Fig. 2). Normally this results in a mode where the sample is impinged with a set of parallel beams which are rocked back and forth, rather than a focused spot which is rastered. This typically yields a Pseudo Kikuchi pattern (see Fig. 3) which contains crystallographic information such as crystal orientation, d-spacing and crystal system.

However, if you place the sample at a different place in space, i.e. not at the place where the parallel beam is rocked, but rather at a very long working distance, one can then strongly excite the objective lens to reconverge the parallel (or divergent) rays and use the second condenser lens as the "focus" control for imaging.

Even if the sample is placed in the bottom of the specimen chamber, with no $X, Y$, or $Z$ translation, the $X$ and $Y$ electronic image shifts, which correspond to the ECP centering coils, allow you to see an $80 \mathrm{~mm}$ by 80 $\mathrm{mm}$ sample and more to specific areas within the $80 \mathrm{~mm}$ square (see Fig's. 4-5).

This helpful technique allows the microscopist to photograph a low magnification "road map" of the sample which is useful in correlating higher magnification SEM images to the appropriate area on the sample without having to remove the sample and use macro photography or low magnification light microscopy.

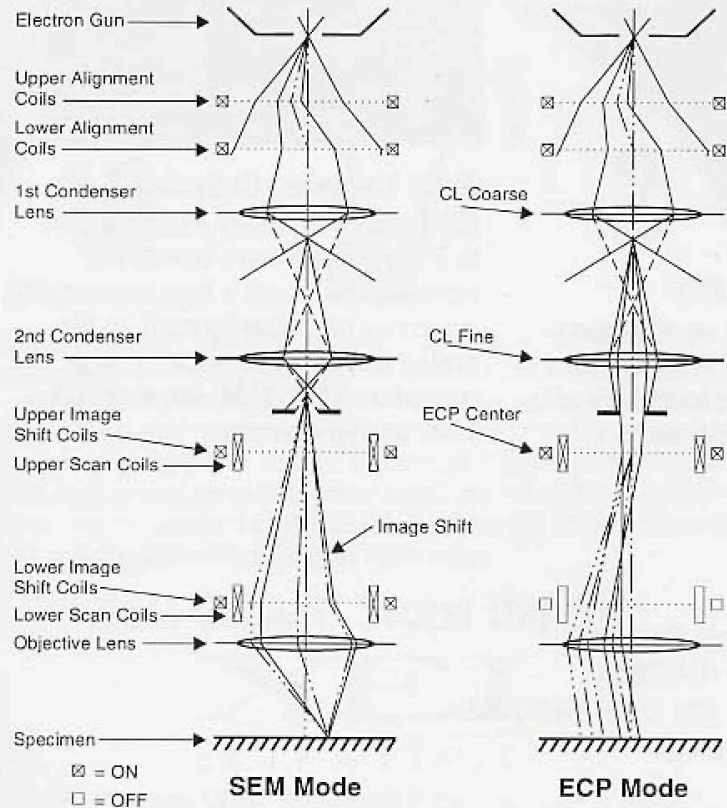

Figure 2: Schematic Ray Diagrams

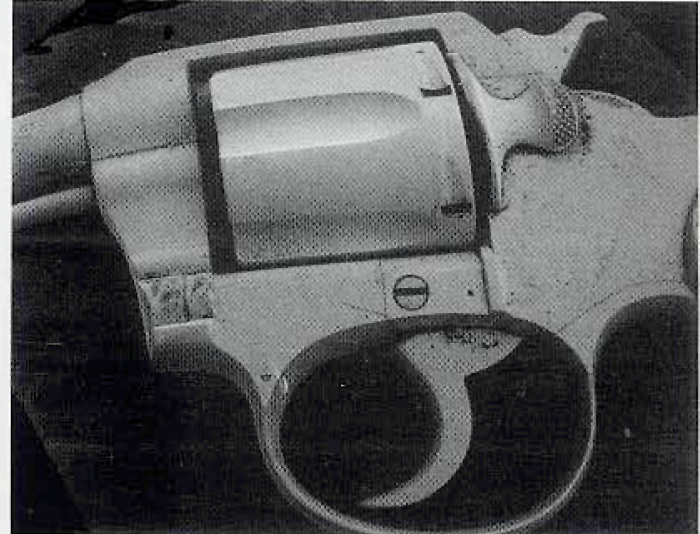

Firgure 1: Ultra Low Magnification (approx. 5X) View of a 32 Caliber Handgun.

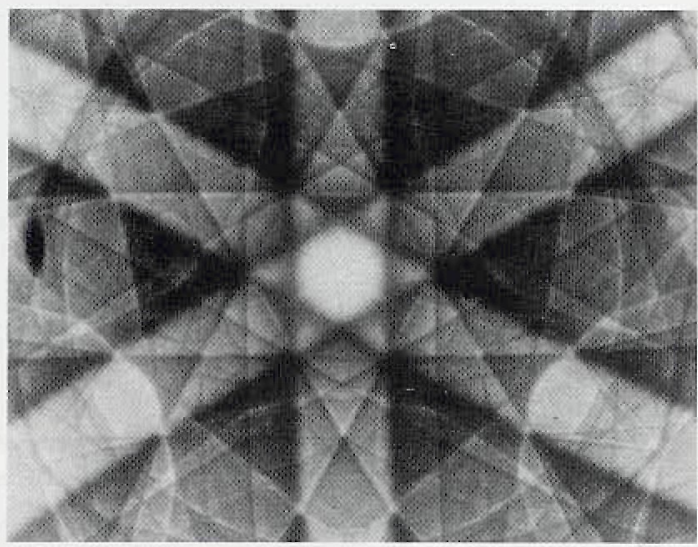

Figure 3: Electron Channeling Pattern of 111 Silicon.

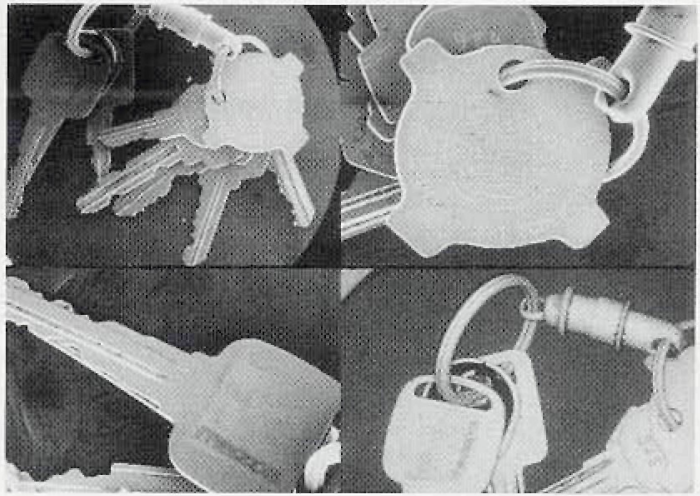

Figure 4: Low magnification views of a set of car keys. Electronic image shift controls were used to view the various areas.

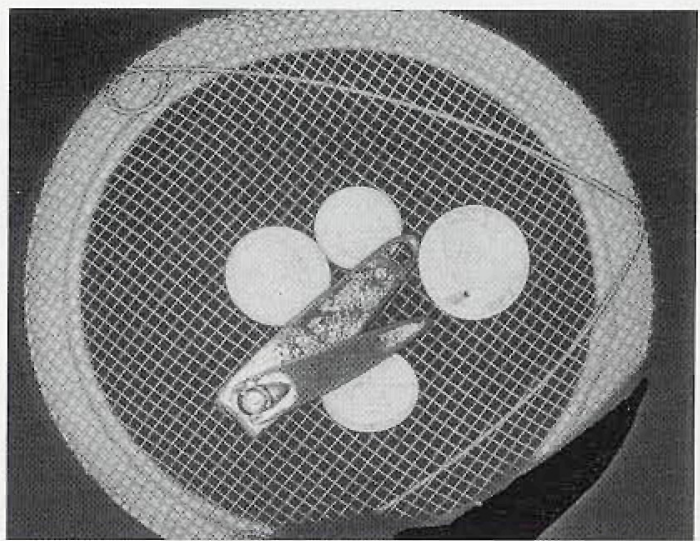

Figure 5: Ultra low magnification $(0.5 \mathrm{X})$ view of pocket contents using backscattered electrons to show atomic number contrast. Note where plating has been worn off the nail clipper. 


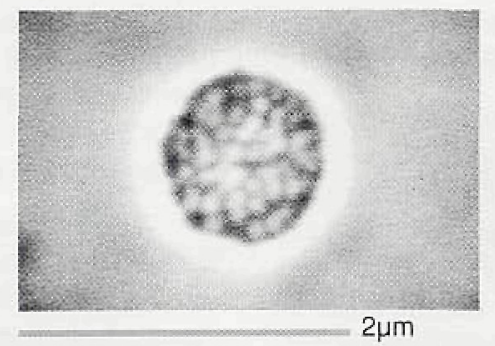

\section{Approximation.}

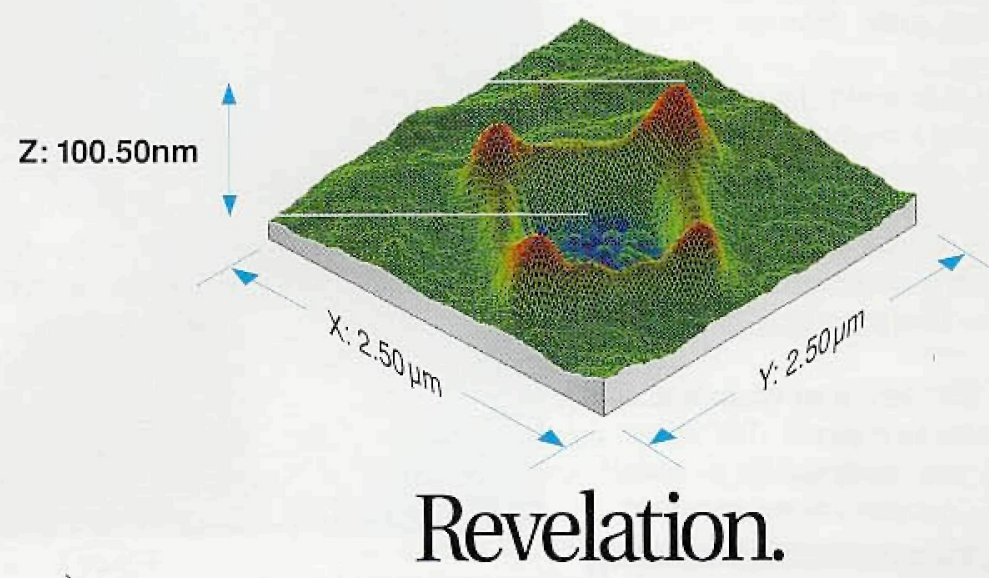

\section{Take the guesswork out of Z-height measurements with our EM accessory.}

Now you can make an inexact science exact. If you have to estimate the Z-height of your samples, then have we got a revelation for you.

Burleigh's EM accessory measures the Z-dimension directly with angstrom accuracy and $5 \%$ precision in the nanometer scale,

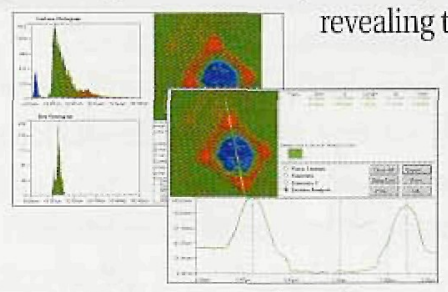

Z-dimensions can be easily measured using the cross-section and surface analysis features of Burleigh's EM accessory.

\begin{abstract}
samples. And this topographical information relates directly to SEM and TEM images, complementing your existing investigation techniques.
\end{abstract}

Tailored for SEM and

TEM users, the Burleigh EM accessory performs 3-D surface analysis under ambient conditionsroutinely, accurately and non-destructively. And its compact design enables you to incorporate it beside your SEM or TEM.

Plus, the Burleigh EM accessory allows you to directly determine surface roughness on samples that are typically problematic for SEMs and TEMs. So you can gain an accurate understanding of all your samples.

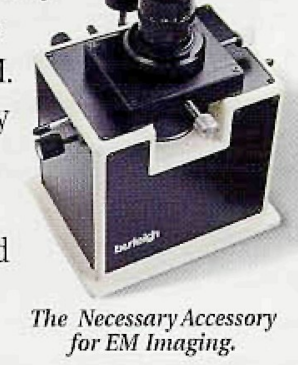

\section{Add depth to your understanding.}

Call us at 1-800-425-8085, ext. 3300, and discover how our EM accessory can provide a revealing new view of your samples. And be sure to inquire about our FREE sample analysis offer. For Europe: (44)01582 766888

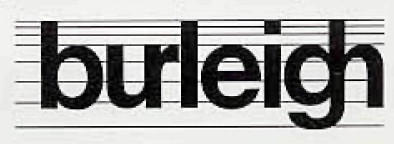

